\title{
Bu open Overactive bladder symptom severity is associated with falls in community- dwelling adults: LOHAS study
}

\author{
Noriaki Kurita, ${ }^{1}$ Shin Yamazaki, ${ }^{1}$ Norio Fukumori, ${ }^{1}$ Kenichi Otoshi, ${ }^{2}$ Koji Otani, ${ }^{2}$ \\ Miho Sekiguchi, ${ }^{2}$ Yoshihiro Onishi, ${ }^{3}$ Misa Takegami, ${ }^{4}$ Rei Ono, ${ }^{5}$ Shigeo Horie, ${ }^{6}$ \\ Shin-ichi Konno, ${ }^{2}$ Shin-ichi Kikuchi, ${ }^{2}$ Shunichi Fukuhara ${ }^{1}$
}

To cite: Kurita N, Yamazaki S, Fukumori N, et al. Overactive bladder symptom severity is associated with falls in community-dwelling adults: LOHAS study. BMJ Open 2013;3:e002413. doi:10.1136/bmjopen-2012002413

- Prepublication history and additional material for this paper are available online. To view these files please visit the journal online (http://dx.doi.org/10.1136/ bmjopen-2012-002413).

Received 27 November 2012 Revised 14 February 2013 Accepted 12 March 2013

This final article is available for use under the terms of the Creative Commons Attribution Non-Commercial 2.0 Licence; see http://bmjopen.bmj.com

For numbered affiliations see end of article.

\section{Correspondence to}

Professor Shunichi Fukuhara; fukuhara.shunichi.6m@kyotou.ac.jp

\section{ABSTRACT}

Objectives: To examine the association between overactive bladder $(O A B)$ symptom severity and falls and the contribution of $O A B$ symptoms to falls in a community-dwelling population.

Design: Cross-sectional study.

Setting: 2 Japanese municipalities.

Participants: A total of 2505 residents aged over 40 years, who participated in health check-ups conducted in 2010. OAB symptom assessed via overactive bladder symptom score (OABSS) was divided into six categories based on distribution and Japanese clinical guidelines. Mobility problems and depressive symptoms were assessed via the Timed Up and Go test and the short form of the Center for Epidemiologic Studies Depression Scale, respectively.

Primary outcome measures: Self-reported any fall and frequent fall $(\geq 2)$ over the 1-month period. Independent contributions to any fall and frequent falls were assessed via logistic regression to generate population-attributable fractions (PAFs), assuming separate causal relationships between $O A B$ symptoms, mobility problems and depressive symptoms and any or frequent falls.

Results: Among the total 1350 participants (mean age: 68.3 years) analysed, any fall and frequent falls were reported by $12.7 \%$ and $4.4 \%$, respectively. Compared with no OABSS score, moderate-to-severe $O A B$ and mild $O A B$ were associated with any fall (adjusted ORs 2.37 (95\% Cl 1.12 to 4.98$)$ and 2.51 (95\% Cl 1.14 to 5.52), respectively). Moderate-tosevere $\mathrm{OAB}$ was also strongly associated with frequent falls (adjusted OR 6.90 (95\% Cl 1.50 to 31.6)). Adjusted PAFs of OAB symptoms were $40.7 \%(95 \% \mathrm{Cl}$ $0.7 \%$ to $64.6 \%)$ for any fall and $67.7 \%(95 \% \mathrm{Cl}$ $-23.1 \%$ to $91.5 \%$ ) for frequent falls. Further, these point estimates were similar to or larger than those of mobility problems and depressive symptoms.

Conclusions: An association does indeed exist between $O A B$ symptom severity and falls, and $O A B$ symptoms might be important contributors to falls among community-dwelling adults. Further longitudinal studies are warranted to examine whether or not $O A B$ symptoms predict risk of future falls and fall-related injuries.

\section{ARTICLE SUMMARY}

Article focus

- An association has been reported between the mere presence of overactive bladder $(O A B)$ and fall-related injuries in a US telephone-based survey study.

- Whether or not the severity of OAB symptoms is related to an increased likelihood of having falls in community-dwelling individuals and how much the $O A B$ symptom impacts on falls remains unclear.

- We assessed the relationship between $O A B$ symptom severity and falls and estimated the potential contribution of $O A B$ symptoms on falls among community-dwelling individuals, assuming a causal relationship between $\mathrm{OAB}$ symptoms and falls.

\section{Key messages}

- OAB was associated with both any fall and frequent $(\geq 2)$ falls over the 1-month study period in community-dwelling adults.

- An increasing trend was noted between $O A B$ symptom severity and frequent falls over the 1-month period.

- Estimates of adjusted population-attributable fraction of $O A B$ symptoms on falls were similar or larger than those of mobility problems and depressive symptoms.

Strengths and limitations of this study

- Strengths of our study include large sample size, population-based format, adjustment for many potential confounding factors and the robustness of the relationship between OAB symptom severity and falls based on sensitivity analyses.

- However, the following limitations should be considered: the cross-sectional design cannot attribute causality based solely on associations between $O A B$ symptom and falls and the assessment of $O A B$ symptoms based on self-reported questionnaire cannot exclude the presence of other urological disorders.

\section{INTRODUCTION}

Overactive bladder $(\mathrm{OAB})$ is a urological syndrome representing a common public 
health problem worldwide; the overall prevalence of $\mathrm{OAB}$ in adults aged over 40 years was reported to be $12.4 \%$ in Japan and $16.6 \%$ in European countries, with incidence increasing with age. ${ }^{2} \mathrm{OAB}$ is characterised by a primary symptom of urgency, with or without incontinence, usually accompanied by nocturia and increased frequency, ${ }^{3}$ although OAB can be diagnosed even without reports of incontinence or nocturia. Separately, falls are also a serious public health problem, with one-third of community-dwelling individuals aged over 65 years falling at least once annually ${ }^{4}$ and with $5-10 \%$ of falls resulting in fracture, head injuries or other serious injuries. ${ }^{5}$ While studies examining incontinence as a risk factor for falls have been well investigated, ${ }^{67}$ few have examined the relationship between $\mathrm{OAB}$ and falls, despite reported overlaps in symptoms between incontinence and $\mathrm{OAB}^{8}{ }^{8}$

While findings from one US telephone survey suggested an association between $\mathrm{OAB}$ and an increased likelihood of being injured in a fall in the previous year among adults aged over 18 years, ${ }^{9}$ whether or not the degree of $O A B$ symptom severity is related to the frequency of falls among community-dwelling older adults over a certain period of time is unclear. This previous study also failed to examine the extent to which $\mathrm{OAB}$ symptoms contribute to falls. Clarification of this potential influence of $\mathrm{OAB}$ symptom severity on falls may help health policy-makers decide how much attention $\mathrm{OAB}$ symptoms should receive to prevent falls among community-dwelling individuals.

Here, we investigated the relationship between $\mathrm{OAB}$ symptom severity and frequency of falls over a 1-month period using data from a large community-dwelling population. We also assessed the potential contribution of $\mathrm{OAB}$ symptom severity to falls in the population.

\section{METHOD}

Our cross-sectional study of the locomotive syndrome and health outcome in Aizu cohort study (LOHAS) which aims to evaluate the risk of cardiovascular disease, quality of life, medical costs and mortality attributable to locomotive dysfunction ${ }^{10}$-was approved by the Research Ethics Committee of Fukushima Medical University School of Medicine. Locomotive syndrome is conceptualised as a condition characterised by a set of associated symptoms due to problems of the locomotive systems. ${ }^{11}$ The locations involved are located in valleys surrounded by mountains, and the main industry in the region is agriculture. Our target population comprised the general population of two communities (Tadami and Minamiaizu Towns) in Fukushima Prefecture, Japan. Eligibility criteria were 'aged over 40 years' and 'participated in annual health check-ups conducted in $2010^{\prime} .{ }^{10}$ Details of the design of the LOHAS have been reported previously. ${ }^{10}$
OAB symptoms, mobility problems and depressive symptoms

$\mathrm{OAB}$ symptoms were the main exposure and assessed based on the OAB Symptom Score (OABSS), ${ }^{12}$ which is designed to quantify $\mathrm{OAB}$ symptoms over the previous week based on four items: daytime frequency, nighttime frequency, urgency and urgency incontinence (see online supplementary table S1). The OABSS has been psychometrically validated and has demonstrated reliability, discriminant validity and responsiveness among patients with OAB. ${ }^{12}{ }^{13}$ Able to be used as an alternative to a bladder diary, ${ }^{14}$ which is considered the gold standard tool for the assessment of OAB symptoms, the OABSS is recommended in Japanese clinical practice. ${ }^{15}$ A total OABSS score of 3 or more and an urgency score of 2 or more is the recommended cut-off for diagnosing $\mathrm{OAB}$, and the severity is further divided into mild (total score of 3-5 points), moderate (6-11 points) and severe (12 or more points), according to Japanese clinical practice guidelines. ${ }^{15}$ Therefore, individuals with a total OABSS score of 2 or less or an urgency score of 1 or less are considered to be 'non-OAB'. To assess the dose-response relationship of the strength of the association between $\mathrm{OAB}$ symptom severity and falls citing non-OAB individuals with a total score of 0 as reference, participants with and without $\mathrm{OAB}$ were divided into six categories based on the severity of total OABSS score and the distribution of the participants: non-OAB with $0,1,2$ or 3 or more points; mild $\mathrm{OAB}$; and moderate-to-severe $\mathrm{OAB}$. The median and range of total OABSS score among the six groups are described in table 1, along with the number of participants.

In addition to accounting for the contribution of $\mathrm{OAB}$ symptoms to falls, we considered the contributions of mobility problems (ie, problems in gait, balance and mobility) and depressive symptoms as well, citing these parameters as secondary exposures in light of their prevalence in ageing societies and their predictability of fall risk in the literature. ${ }^{6} 1617$ Mobility problems were assessed using the Timed Up and Go (TUG) test, in which the individuals are timed with a stopwatch when rising from a chair, walking $3 \mathrm{~m}$ and turning to return to sit on the chair. The TUG test is considered to be a reflection of function in gait, balance and mobility ${ }^{17}$ and is commonly used among older persons in geriatric clinics to evaluate physical performance, ${ }^{18}$ with a greater score indicating more mobility problems. Given the findings from a meta-analysis suggesting that reference TUG values differ by age group (mean=8.1 $\mathrm{s}$ for 60 to $69,9.2 \mathrm{~s}$ for 70 to 79 and $11.3 \mathrm{~s}$ for 80 to 99 -year-olds), ${ }^{19}$ specifying a uniform cut-off of TUG value to discriminate the presence of mobility problems was difficult, and the TUG value was therefore treated as a continuous variable. Although no reference TUG values have been established for persons aged 40-59 years, we believe that ' $5.5-8.1 \mathrm{~s}$ ' is a feasible range, as a previous study 
Table 1 Baseline characteristics of the analysis population

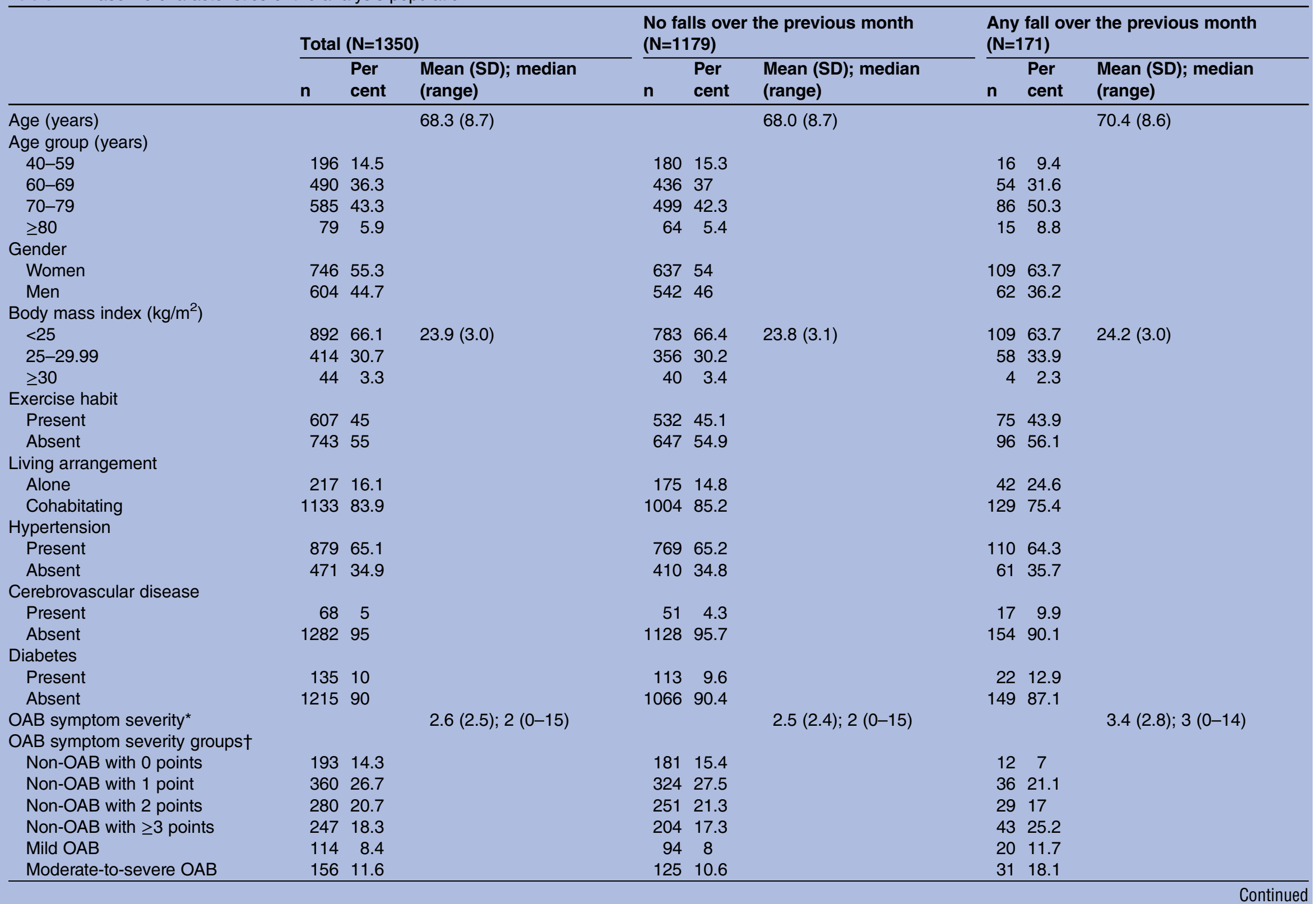




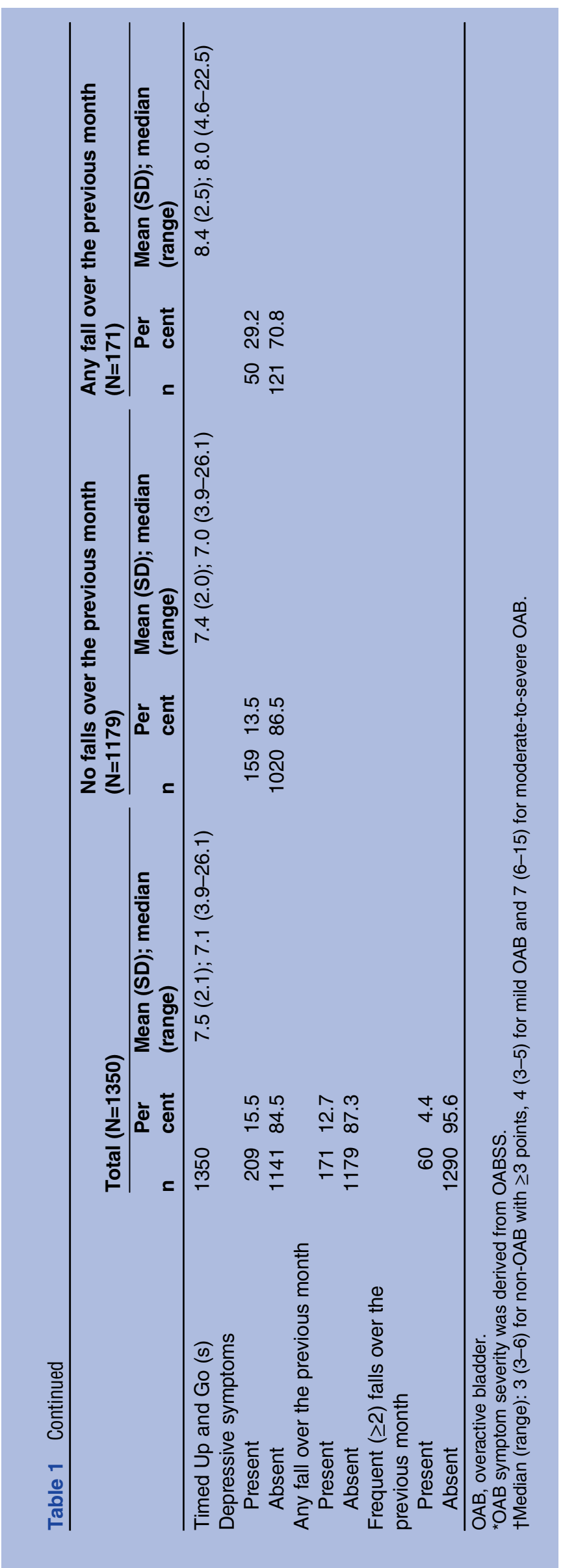

found the mean TUG value to be $5.5 \mathrm{~s}$ in healthy young adults (mean age: 22.3 years) the mean TUG value in adults aged 60-69 years was $8.1 \mathrm{~s}$ as described above. $^{20}$

Depressive symptoms were assessed using the 10-item version of the Center for Epidemiological Studies Depression Screening Index (CES-D), which is designed to quantify the number and frequency of symptoms of depression. Depressive mood was defined as a score of 10 or greater. ${ }^{21}$

\section{Clinical outcomes}

We examined two clinical outcomes of interest-'any fall over a 1-month period' and 'frequent $(\geq 2)$ falls over a 1-month period'-based on subjects' answers to the question, 'Over the past year, have you fallen down?' to establish fall history. Participants who responded, 'Yes', were then asked the follow-up question, 'How many times have you fallen down over the past month?' with responses of 'zero', 'once', 'twice' or 'three or more times' allowed. Those who reported at least 'once' were considered to have had 'any fall' over the previous month. Those who reported at least 'twice' $(\geq 2)$ were, in addition to 'any fall', further considered to have had 'frequent falls'.

\section{Measurement of potential confounding variables}

Potential confounding variables examined in the present study were sociodemographic characteristics including age, gender, exercise habit and living arrangement (alone or cohabitating), as well as the presence of cerebrovascular disease, all obtained via self-reported questionnaire; body mass index and blood pressure, as measured by local nurse practitioners; hypertension, defined as systolic blood pressure $\geq 140 \mathrm{~mm} \mathrm{Hg}$ and diastolic blood pressure $\geq 90 \mathrm{~mm} \mathrm{Hg}$ or by individuals reporting attending a physician for treatment; and diabetes, defined as having glycosylated haemoglobin values $\geq 6.1 \%$, as described by the Japanese Diabetes Society (equivalent to $\geq 6.5 \%$ described in National Glycohemoglobin Standardization Program (NGSP) values $^{22}$ ) or by individuals reporting attending a physician for treatment. Individuals were considered to engage in exercise if they answered 'Yes' to the question concerning whether or not they had participated in moderate physical activity (making the individual breathe somewhat harder than normal and including situations such as carrying light loads, bicycling at a regular pace or doubles tennis) in the previous 7 days.

\section{Statistical analysis}

Participants with complete data were entered into the primary analyses. Statistical analyses were conducted using Stata V.11.0 (Stata Corp., College Station, Texas, USA). Sociodemographic characteristics, comorbidities, main (OAB symptom severity) and secondary exposures (TUG values and depressive symptoms) and outcomes (any fall and frequent falls over the previous month) were described. 
Effect measures in the present study were ORs of the three exposures for any fall and frequent falls estimated using logistic regression models, with separate models applied for any fall and frequent falls over the previous month. To estimate adjusted ORs, the potential confounding variables described above were simultaneously forced into the models along with the three exposures. The goodness of fit of the models was assessed using the Hosmer-Lemeshow test.

The potential effect modification of gender difference, TUG and depressive symptoms on the association between $\mathrm{OAB}$ symptom severity and any fall or frequent falls over the previous month was tested by adding three interaction pairs (the product terms of OAB symptoms severity with gender, TUG or depressive symptoms) to the logistic regression models.

In addition, using the regression models based on a previously reported method, a test of linear trends across six OAB symptom severity categories was performed by treating the categories as a continuous variable using their median values. ${ }^{23}$ To estimate the potential contribution of each of the exposures (OAB symptom severity, mobility problems or depression symptoms) to falls, we computed population-attributable fractions (PAFs). In general, in a cross-sectional study, PAF is the fraction of the prevalent outcomes that would be prevented if a certain exposure was removed. In other words, if the exposure was removed, then some fraction of the outcomes could be prevented, and that fraction is PAF. For this study, outcomes used were both 'any fall' and 'frequent falls', and exposures used were OAB symptom severity, mobility problems and depression symptoms. One assumption of this computation is that there is an unconfounded, causal relation between exposure(s) and outcome(s). PAFs were computed with the logistic regression models and the user-written command 'punaf' in Stata V.11.0. ${ }^{24} 25$

We also conducted sensitivity analyses by imputing missing covariate values. A multiple imputation approach using a chained equations method was used, ${ }^{26} 27$ with results showing that 1037 participants (41.9\%) had missing covariate values. $\mathrm{p}<0.05$ was considered statistically significant.

\section{RESULTS}

Of the 2505 participants in the health check-up (figure 1), $2387(95.3 \%)$ had both exposure and outcome variables. After excluding 1037 participants with at least one confounding variables missing, the remaining 1350 participants were ultimately entered into the primary analyses.

Baseline characteristics are presented in table 1. Participants tended to be older, with $85.5 \%$ aged over 60 years. The prevalence of hypertension and diabetes were $65.1 \%$ and $10.0 \%$, respectively. The prevalence of $\mathrm{OAB}$ which met the criteria described in the Japanese clinical guidelines was $20.0 \%$, with prevalence of moderate-to-severe $\mathrm{OAB}$ at $11.6 \%$. Of note, the prevalence of non-OAB individuals with $\geq 3$ points was $18.3 \%$. Age-stratified prevalence of OAB in our participants was $9.7 \%$ for 40 to $59,14.7 \%$ for 60 to $69,27.0 \%$ for 70 to 79 and $26.6 \%$ for 80 to 89 -year-olds, values similar to those noted in a previous study in Japan. ${ }^{2}$ Mean TUG time in the present study was $7.5 \mathrm{~s}$ (SD $2.1 \mathrm{~s}$ ), ranging from a minimum of $3.9 \mathrm{~s}$ to a maximum of $26.1 \mathrm{~s}$. Age-stratified mean TUG values were $6.4 \mathrm{~s}$ for 40 to 59 , $6.8 \mathrm{~s}$ for 60 to $69,8.2 \mathrm{~s}$ for 70 to 79 and $9.8 \mathrm{~s}$ for 80 to 89-year-olds, values which were smaller than the reported reference. ${ }^{19}$ The prevalence of depressive symptoms was $15.5 \%$. The prevalence of any fall and frequent falls over the previous month was $12.7 \%$ and $4.4 \%$, respectively, while age-stratified prevalence of any fall and frequent falls over the previous month were $8.2 \%$ and $2.0 \%$ for 40 to $59,11.0 \%$ and $3.3 \%$ for 60 to $69,14.7 \%$ and $5.6 \%$ for 70 to 79 , and $19.0 \%$ and $8.9 \%$ for 80 to 89-year-olds, respectively.

Sociodemographic characteristics were similar between participants with and without missing confounding variables (see online supplementary table S2)

Figure 1 Flow chart of the study participants.

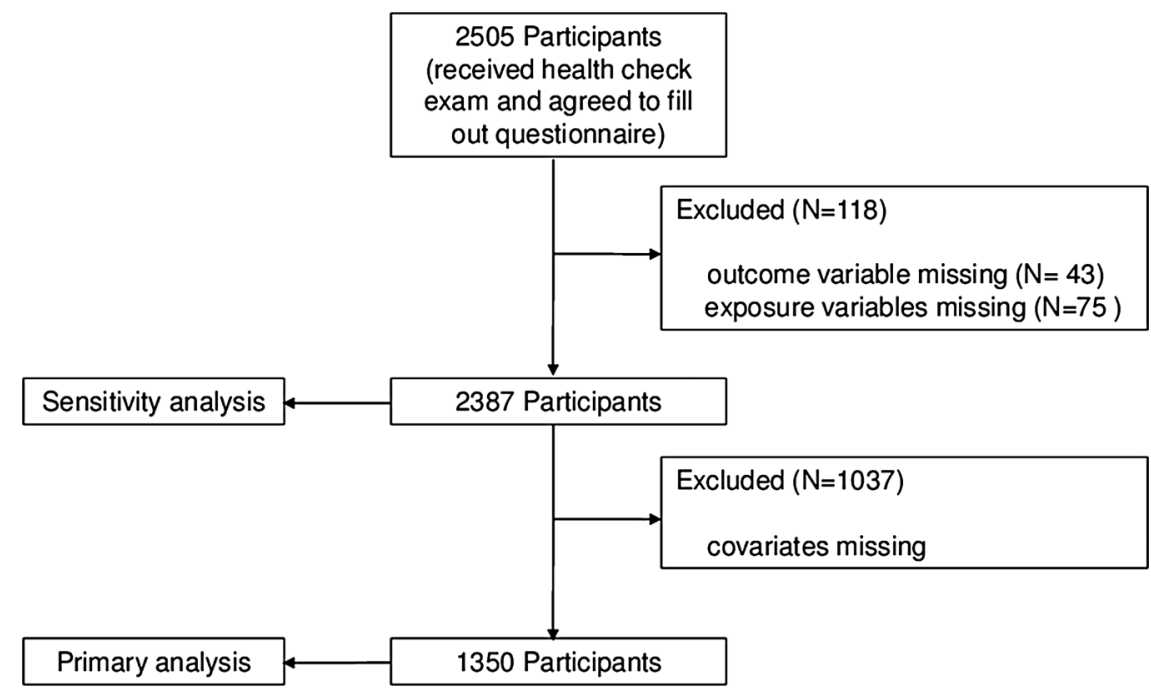


except for gender, as men were more likely to have missing variables. As a group, their OAB symptom severity, TUG value, proportion with depressive symptoms and proportions of any and frequent falls over the 1-month period examined were similar.

In crude analyses, the presence of $\mathrm{OAB}$, increase in TUG, and the presence of depressive symptom indicated a greater likelihood of having both any and frequent falls (table 2), and ORs for these parameters were attenuated in adjusted analyses. Participants with moderate-to-severe $\mathrm{OAB}$ had a greater likelihood of having any fall and frequent fall than non-OAB individuals with 0 points (adjusted OR for any fall=2.37 (95\% CI 1.12 to 4.98); and adjusted OR for frequent fall $=6.90$ (95\% CI 1.50 to 31.6)). In addition, those with mild $\mathrm{OAB}$ and non-OAB individuals with $\geq 3$ points had a similarly greater likelihood of having any fall (adjusted OR 2.51 (95\% CI 1.14 to 5.52), and adjusted $\mathrm{OR}=2.46$ (95\% CI 1.23 to 4.92 ), respectively) than non-OAB individuals with 0 points. We noted no significant trends in likelihood of any fall across $\mathrm{OAB}$ severity categories ( $\mathrm{p}=0.062)$, presumably because of similar strength of likelihoods among the top three categories of $\mathrm{OAB}$ severity. In contrast, we noted a significant increasing trend in the likelihood of frequent fall across $\mathrm{OAB}$ severity categories $(\mathrm{p}=0.015)$.

The adjusted ORs of TUG for any and frequent falls were 1.25 (95\% CI 1.06 to 1.47 ) and 1.36 (95\% CI 1.09 to 1.70), respectively, for each increase in SD of TUG, suggesting that the increase in TUG was associated with these outcomes. The adjusted ORs of depression for any and frequent falls were 1.96 (95\% CI 1.31 to 2.94) and $1.98(95 \%$ CI 1.06 to 3.71$)$, respectively. The goodness-of-fit values of the covariate-adjusted logistic regression models for any fall and frequent falls were 0.217 and 0.364 , respectively.

Overall, effect modification by gender difference, TUG and depressive symptoms on the association between the $\mathrm{OAB}$ severity and any fall or frequent falls was not apparent (data not shown). Therefore, PAF analyses and sensitivity analyses were performed without considering the effect modification. Analyses results indicated that, if $\mathrm{OAB}$ symptoms could have been reduced to OABSS 0 points (with all other parameters remaining the same), a sizeable proportion of any and frequent falls could have been prevented potentially (40.7\% (95\% CI $0.7 \%$ to $64.6 \%$ ) and $67.7 \%$ (95\% CI $-23.1 \%$ to $91.5 \%$ ), respectively), although of note-the lower CI of the proportion for frequent falls was already below $0 \%$ (table 3). Similarly, if TUG could have been improved up to $4 \mathrm{~s}$ (the second smallest value in this population), the estimated proportions of the any and frequent falls that could potentially have been prevented were $30.4 \%$ (95\% CI $7.6 \%$ to $47.6 \%)$ and $45.8 \%(95 \%$ CI $13.1 \%$ to $66.2 \%$ ), respectively. As for depressive symptoms, these estimated proportions were smaller, at $11.7 \%$ (95\% CI $3.8 \%$ to $18.9 \%)$ and $15.3 \%(95 \% \mathrm{CI}-1.3 \%$ to $29.1 \%$ ) for any and frequent falls, respectively.

Table 2 Associations between OAB symptom severity, mobility problems and depressive symptoms and falls

\begin{tabular}{|c|c|c|c|c|c|}
\hline & \multirow[b]{2}{*}{$\begin{array}{l}\text { Number of } \\
\text { participants }\end{array}$} & \multicolumn{2}{|c|}{ Any fall over the previous month } & \multicolumn{2}{|c|}{$\begin{array}{l}\text { Frequent }(\geq 2) \text { falls over the previous } \\
\text { month }\end{array}$} \\
\hline & & $\begin{array}{l}\text { Unadjusted OR } \\
(95 \% \mathrm{Cl})\end{array}$ & $\begin{array}{l}\text { Adjusted* OR } \\
(95 \% \mathrm{CI})\end{array}$ & $\begin{array}{l}\text { Unadjusted OR } \\
(95 \% \mathrm{Cl})\end{array}$ & $\begin{array}{l}\text { Adjusted* OR } \\
(95 \% \mathrm{Cl})\end{array}$ \\
\hline \multicolumn{6}{|l|}{ OAB symptom } \\
\hline $\begin{array}{l}\text { Non-OAB with } 0 \\
\text { points }\end{array}$ & 193 & 1 & 1 & 1 & 1 \\
\hline $\begin{array}{l}\text { Non-OAB with } 1 \\
\text { point }\end{array}$ & 360 & 1.68 (0.85 to 3.30$)$ & $1.61(0.81$ to 3.21$)$ & $3.29(0.73$ to 14.9$)$ & $2.97(0.65$ to 13.6$)$ \\
\hline $\begin{array}{l}\text { Non-OAB with } 2 \\
\text { points }\end{array}$ & 280 & $1.74(0.87$ to 3.51$)$ & $1.46(0.71$ to 2.99$)$ & 3.17 (0.68 to 14.8$)$ & $2.17(0.45$ to 10.4$)$ \\
\hline $\begin{array}{l}\text { Non-OAB with } \geq 3 \\
\text { points }\end{array}$ & 247 & 3.18 (1.63 to 6.22$)$ & 2.46 (1.23 to 4.92$)$ & 4.88 (1.08 to 22.1$)$ & $3.35(0.72$ to 15.6$)$ \\
\hline Mild OAB & 114 & $3.21(1.50$ to 6.85$)$ & 2.51 (1.14 to 5.52$)$ & 6.25 (1.28 to 30.6$)$ & $4.30(0.84$ to 22.0$)$ \\
\hline \multicolumn{6}{|l|}{$\mathrm{OAB}$} \\
\hline & & Trend $p=0.018$ & Trend $p=0.062$ & Trend $p=0.006$ & Trend $p=0.015$ \\
\hline \multicolumn{6}{|l|}{ Timed Up and Go } \\
\hline Per $1 \mathrm{~s}$ & 1350 & $1.21(1.13$ to 1.29$)$ & $1.11(1.03$ to 1.20$)$ & $1.28(1.17$ to 1.39$)$ & $1.16(1.04$ to 1.29$)$ \\
\hline Per 1SD & & $1.49(1.30$ to 1.71$)$ & 1.25 (1.06 to 1.47$)$ & 1.67 (1.39 to 2.00$)$ & $1.36(1.09$ to 1.70$)$ \\
\hline \multicolumn{6}{|l|}{ Depressive symptoms } \\
\hline Absent & 1141 & 1 & 1 & 1 & 1 \\
\hline Present & 209 & 2.65 (1.83 to 3.84$)$ & 1.96 (1.31 to 2.94$)$ & 3.16 (1.82 to 5.48$)$ & $1.98(1.06$ to 3.71$)$ \\
\hline
\end{tabular}


Table 3 Population-attributable fraction for falls *

\begin{tabular}{|c|c|c|}
\hline & $\begin{array}{l}\text { Any fall over the previous month } \\
\text { Estimates }(95 \% \mathrm{Cl})\end{array}$ & $\begin{array}{l}\text { Frequent }(\geq 2) \text { falls over the previous month } \\
\text { Estimates }(95 \% \mathrm{Cl})\end{array}$ \\
\hline OAB symptom & $40.7 \%(0.7 \%$ to $64.6 \%)$ & $67.7 \%(-23.1 \%$ to $91.5 \%)$ \\
\hline Timed Up and $\mathrm{Go}^{\dagger}$ & $30.4 \%(7.6 \%$ to $47.6 \%)$ & $45.8 \%(13.1 \%$ to $66.2 \%)$ \\
\hline Depressive symptoms & $11.7 \%$ (3.8\% to $18.9 \%)$ & $15.3 \%(-1.3 \%$ to $29.1 \%)$ \\
\hline
\end{tabular}

In sensitivity analyses using multiply imputed data for 2387 participants, similar associations were noted between OAB severity symptoms and increased ORs for both clinical outcomes (see online supplementary table S3).

\section{DISCUSSION}

In this large, cross-sectional study of community-dwelling adults, moderate-to-severe $\mathrm{OAB}$ and mild $\mathrm{OAB}$ were both associated with any falls over the 1-month study period. We also observed an increasing trend in OAB symptom severity with frequent falls, and moderate-to-severe $\mathrm{OAB}$ was strongly associated with frequent falls. In addition, $\mathrm{OAB}$ symptoms were found to likely experience similar or greater impact on falls than mobility problems and depressive symptoms. These findings may encourage health policy-makers and physicians to pay more attention to $\mathrm{OAB}$ symptoms than at present to help prevent falls among community-dwelling adults.

Our findings here agree well with those of a previous study, which showed that the presence of OAB increased the likelihood of being injured from a fall in the previous year (OR 2.26). ${ }^{9}$ However, the present and previous studies differed in several respects. First, the mean age of the participants in our study was much higher than that in the previous study (68.3 vs 54.2 years), suggesting that our findings will be more applicable in ageing societies. Second, the previous study used matching to compare subjects with and without OAB; as such, their study design limited the accurate determination of prevalence of OAB symptom severity and estimation of PAF. Our use of PAF in the present study suggested the potential magnitude of reduction in falls that could theoretically be prevented in an ageing society such as the one examined here. PAF estimates of OAB symptoms in societies such as that involved in the present study with an unignorable proportion of falls would be of significant relevance to health policy-makers and physicians. Third, the telephone-based survey method hampered the determination of several biological measures in the previous study, such as body mass index and mobility problems (ie, TUG), which are potentially confounding variables that should be considered in the accurate assessment of the relationship between $\mathrm{OAB}$ and falls. Fourth, the dose-response relationship between OAB symptom severity and falls was not evaluated in the previous study; therefore, we feel that the logical connection between $\mathrm{OAB}$ and falls in the previous study is less convincing than that in the present study.

Several strengths to the present study warrant mention. First, we demonstrated the relationship between $\mathrm{OAB}$ symptom severity and falls in a large, community-dwelling population, adjusting for confounding variables such as body mass index and depressive symptoms, which are potentially related to both OAB and falls. Second, we showed that the contribution of OAB symptoms to falls might be similar or larger than that of mobility problems and depressive symptoms, according to PAF estimates. These findings provide a basis for considering the effective use of limited medical resources to prevent falls. Noted similarities in the relationship of OAB symptom severity and falls in our sensitivity analyses indicate that our results are robust.

However, several limitations to the present study also warrant mention. First, we were unable to include the medication usage and all chronic conditions in our analyses; given that use of antihypertensives or sedatives are also potential risk factors for falls, the association between $\mathrm{OAB}$ symptom severity and falls in the present study might be confounded by these factors. To mitigate this limitation, we adjusted for individuals with hypertensive problems. Effects of depressive symptoms on falls might be mediated partly by sedatives, as individuals with depressive symptoms are often prescribed sedatives to treat depressive emotion or sleep problems. However, we believe it unlikely that effects of OAB on falls are confounded by sedatives. While some specialists may express concern about whether or not antimuscarinics for $\mathrm{OAB}$ are associated with an elevated risk of falls among treated individuals, to date, little evidence has been generated which supports such an association. ${ }^{28}$ Cognitive impairment and arthritis (including osteoarthritis) are also potential risk factors for falls ${ }^{29} 30$; however, individuals with cognitive impairment are unlikely to participate in epidemiological studies and complete selfreported questionnaires. ${ }^{31}$ Regarding arthritis in particular, its burden on functional mobility should be reflected by TUG. As such, we believe that these confounding factors had negligible effects on our analyses. Second, given that the definition of $\mathrm{OAB}$ in this study used the criteria defined by the Japanese Urological Associations, ${ }^{15}$ the presence of other urological 
disorders (such as benign prostatic enlargement and urinary tract infection) cannot be ruled out. Third, the cross-sectional design of the present study means that we cannot attribute causality from the associations between any exposures and falls. While we believe that $\mathrm{OAB}$ being a cause of falling is possible and biologically plausible, some associations such as those between depressive symptoms and falls can be explained by reverse causality. Fourth, although sensitivity analyses using $95.2 \%$ of the participants in the health check-up exam showed an association similar to that noted in primary analyses between OAB symptom severity and falls, a high rate of missing data was noted in the primary analyses. Fifth, $\mathrm{PAF}$ of $\mathrm{OAB}$ symptom for frequent falls had low precision, although its point estimate suggested that a sizeable proportion of frequent falls might be preventable. Further, we were unable to estimate PAFs in the sensitivity analysis because, at present, statistical analyses are difficult to conduct using multiply imputed data. However, distributions of OAB symptom severity, TUG and depressive symptoms are similar between individuals with complete data and those with missing covariates. Therefore, it is unlikely that PAF estimates in the complete dataset would be starkly different from those in the target population.

We feel that the present findings will influence the activities of physicians and health policy-makers for several reasons. First, $\mathrm{OAB}$ symptoms are potentially modifiable risk factors for falls, able to be managed with a combination of behavioural therapy and antimuscarinics indicated for OAB. Indeed, well-designed clinical trials have shown that antimuscarinics such as solifenacin and tolterodine reduce the severity of OAB symptoms by more than $60 \% .{ }^{15} \mathrm{~A}$ further study is needed to clarify whether or not the treatment of $\mathrm{OAB}$ symptoms is associated with the reduction of falls. However, community-dwelling individuals may hesitate to consult physicians regarding $\mathrm{OAB}$ symptoms, in light of potential stigma attached to the disorder. ${ }^{9}$ As such, when physicians encounter patients who have suffered falls, they should be sensitive when asking patients about their experiencing any $\mathrm{OAB}$ symptoms. This communication is applicable to both genders, as no gender differences were apparent in the relationship between OAB symptoms and falls. Second, the dose-response relationship between $\mathrm{OAB}$ symptom severity and frequent falls suggests that not only nocturia and incontinence but also urgency and frequency may contribute to the risk of fall. While previous fall-related studies have thus far focused primarily on nocturia and incontinence, ${ }^{6}{ }^{32}$ more frequent walking to the bathroom may raise the risk of falling. In addition, urgency may cause individuals to lose focus while walking due to nocturia or frequency, rendering them to be more susceptible to falling. Third, given the large PAF values for OAB symptoms for falls in our study, health policy-makers and physicians should consider $\mathrm{OAB}$ to be not only a mere syndrome reducing the health-related quality of life but also a large contributor to falls among community-dwelling individuals. As such, screening for OAB at health check-ups and appropriate referral may be a reasonable health plan to interact. We should also point out that physicians caring for fall victims and those treating $\mathrm{OAB}$ patients are usually different and may therefore not be communicating well. Compared with these physicians, primary care physicians and their team may provide better and care for the symptoms of the individuals.

In conclusion, $\mathrm{OAB}$ symptom severity in communitydwelling adults was found to be associated with any and frequent falls over a 1-month period. Further, OAB symptoms might have a similar or greater contribution to falls than mobility problems or depressive symptoms, both common in ageing societies. Further longitudinal studies are warranted to examine whether or not $\mathrm{OAB}$ symptoms predict future falls and fall-related injuries.

\section{Author affiliations}

${ }^{1}$ Department of Healthcare Epidemiology, Graduate School of Medicine and Public Health, Kyoto University, Kyoto, Japan

${ }^{2}$ Department of Orthopedic Surgery, Fukushima Medical University School of Medicine, Fukushima, Japan

${ }^{3}$ Institute for Health Outcomes \& Process Evaluation Research (i-Hope international), Kyoto, Japan

${ }^{4}$ Department of Preventive Medicine and Epidemiologic Informatics, National Cerebral and Cardiovascular Center, Osaka, Japan

${ }^{5}$ Department of Community Health Sciences, Kobe University Graduate School of Health Sciences, Kobe, Japan

${ }^{6}$ Department of Urology, Juntendo University School of Medicine, Tokyo, Japan

Contributors NK had the idea for the study, participated in its design, performed the statistical analyses and drafted the manuscript. NF, $\mathrm{KeO}, \mathrm{KoO}$, MS, MT and RO participated in acquiring the data and helped in analysing the data. SY, YO, SKO, SKi are coordinators of the locomotive syndrome and health outcome in Aizu cohort study and participated in critical review of the manuscript. SH participated in the interpretation of the data and critical review of the manuscript. SF had the idea for the study and participated in the design of the study, interpretation of the data and critical reviewing and editing of the manuscript.

Funding This research received no specific grant from any funding agency in the public, commercial or not-for-profit sectors.

\section{Competing interests None.}

Ethics approval The locomotive syndrome and health outcome in Aizu cohort study was approved by the Research Ethics Committee of Fukushima Medical University School of Medicine.

Provenance and peer review Not commissioned; externally peer reviewed.

Data sharing statement No additional data are available.

\section{REFERENCES}

1. Milsom I, Abrams P, Cardozo L, et al. How widespread are the symptoms of an overactive bladder and how are they managed? A population-based prevalence study. BJU Int 2001;87:760-66.

2. Homma $Y$, Yamaguchi $\mathrm{O}$, Hayashi $\mathrm{K}$, et al. An epidemiological survey of overactive bladder symptoms in Japan. BJU Int 2005;96:1314-18.

3. Abrams $P$, Cardozo L, Fall M, et al. The standardisation of terminology in lower urinary tract function: report from the standardisation sub-committee of the International Continence Society. Urology 2003;61:37-49.

4. Tinetti ME, Speechley M, Ginter SF. Risk factors for falls among elderly persons living in the community. $N$ Engl $J$ Med 1988;319:1701-7. 
5. Rubenstein LZ, Josephson KR. The epidemiology of falls and syncope. Clin Geriatr Med 2002;18:141-58.

6. Tromp AM, Smit JH, Deeg DJH, et al. Predictors for falls and fractures in the Longitudinal Aging Study Amsterdam. J Bone Miner Res 1998;13:1932-9.

7. Brown JS, McGhan WF, Chokroverty S. Comorbidities associated with overactive bladder. Am J Manag Care 2000;6:S574-9.

8. Irwin DE, Milsom I, Hunskaar S, et al. Population-based survey of urinary incontinence, overactive bladder, and other lower urinary tract symptoms in five countries: results of the EPIC study. Eur Urol 2006;50:1306-15.

9. Wagner TH, Hu TW, Bentkover J, et al. Health-related consequences of overactive bladder. Am J Manag Care 2002;8: S598-607.

10. Otani K, Takegami M, Fukumori N, et al. Locomotor dysfunction and risk of cardiovascular disease, quality of life, and medical costs: design of the locomotive syndrome and health outcome in Aizu Cohort Study (LOHAS) and baseline characteristics of the study population. J Orthop Sci 2012;17:261-71.

11. Nakamura K. Locomotive syndrome: disability-free life expectancy and locomotive organ health in a 'super-aged' society. J Orthop Sci 2009;14:1-2.

12. Homma Y, Yoshida M, Seki N, et al. Symptom assessment tool for overactive bladder syndrome-overactive bladder symptom score. Urology 2006;68:318-23.

13. Gotoh M, Homma Y, Yokoyama O, et al. Responsiveness and minimal clinically important change in overactive bladder symptom score. Urology 2011;78:768-73.

14. Homma $\mathrm{Y}$, Kakizaki $\mathrm{H}$, Yamaguchi $\mathrm{O}$, et al. Assessment of overactive bladder symptoms: comparison of 3-day bladde diary and the overactive bladder symptoms score. Urology 2011;77:60-4

15. Yamaguchi $O$, Nishizawa $O$, Takeda $M$, et al. Clinical guidelines for overactive bladder. Int J Urol 2009;16:126-42.

16. Snowdon J. Is depression more prevalent in old age? Aust $N Z J$ Psychiatry 2001;35:782-7.

17. van lersel MB, Munneke M, Esselink RAJ, et al. Gait velocity and the Timed-Up-and-Go test were sensitive to changes in mobility in frail elderly patients. J Clin Epidemiol 2008;61:186-91.

18. Podsiadlo D, Richardson S. The timed 'Up \& Go': a test of basic functional mobility for frail elderly persons. J Am Geriatri Soc $1991 ; 39: 142-8$
19. Bohannon RW. Reference values for the Timed Up and Go test: a descriptive meta-analysis. J Geriatr Phys Ther 2006;29:64-8.

20. Brotherton SS, Williams HG, Gossard JL, et al. Are measures employed in the assessment of balance useful for detecting differences among groups that vary by age and disease state? $J$ Geriatr Phys Ther 2005;28:14-19.

21. Andresen EM, Malmgren JA, Carter WB, et al. Screening for depression in well older adults-evaluation of a short-form of the CES-D. Am J Prev Med 1994;10:77-84.

22. Seino $\mathrm{Y}$, Nanjo K, Tajima N, et al. Report of the committee on the classification and diagnostic criteria of diabetes mellitus. J Diabetes Invest 2010;1:212-28.

23. Hu FB, Sigal RJ, Rich-Edwards JW, et al. Walking compared with vigorous physical activity and risk of type 2 diabetes in women: a prospective study. JAMA 1999;282:1433-9.

24. Greenland S, Drescher K. Maximum likelihood estimation of the attributable fraction from logistic models. Biometrics 1993;49:865-72.

25. PUNAF: Stata module to compute population attributable fractions for cohort studies [program]. S457193 version: Boston College Department of Economics, 2010.

26. Rubin D. Multiple Imputation for Nonresponse in Surveys. New York: John Wiley \& Sons, 1987.

27. van Buuren S, Boshuizen HC, Knook DL. Multiple imputation of missing blood pressure covariates in survival analysis. Stat Med 1999;18:681-94.

28. Hunter KF, Wagg A, Kerridge $\mathrm{T}$, et al. Falls risk reduction and treatment of overactive bladder symptoms with antimuscarinic agents: a scoping review. Neurourol Urodyn 2011;30:490-4.

29. Arden NK, Nevitt MC, Lane NE, et al. Osteoarthritis and risk of falls, rates of bone loss, and osteoporotic fractures. Arthritis Rheum 1999;42:1378-85.

30. van Schoor NM, Smit JH, Pluijm SMF, et al. Different cognitive functions in relation to falls among older persons: immediate memory as an independent risk factor for falls. J Clin Epidemiol 2002;55:855-62.

31. Jacomb PA, Jorm AF, Korten AE, et al. Predictors of refusal to participate: a longitudinal health survey of the elderly in Australia. BMC Public Health 2002;2:4

32. Vaughan CP, Brown CJ, Goode PS, et al. The association of nocturia with incident falls in an elderly community-dwelling cohort. Int J Clin Pract 2010;64:577-83. 